\title{
Les Métis et le Québec: scissions dans la sémiosphère fédérée du
}

\section{Canada}

\section{The Metis and Québec: Splits in the federal semiosphere of Canada}

\author{
Licia Soares de Souza ${ }^{1}$
}

Submetido em 16 de março e aprovado em 24 de março de 2017.

Résumé: Les rébellions des Métis du Nord-ouest menées par Louis Riel ont réussi à remettre en cause la position du Québec dans la fédération canadienne. Il y a eu une prise de conscience des différences entre les peuples anglophones et francophones qui n'avait pas été prise en considération lors de l'Acte de l'Amérique du Nord britannique. Dans cet article, nous analysons quelques extraits des éditoriaux du journal La Presse entre février et novembre 1885, photocopiés à partir des microfilms de la bibliothèque de l'Université du Québec à Montréal. Nous constatons que les premiers éditoriaux étaient en symbiose avec l'idée d'une union de la fédération, en ce qu'ils condamnaient la «sauvagerie» des rebelles indiens et métis. Compte tenu de la réaction des lecteurs qui voyaient dans la cause des Métis une lutte semblable à celles que les Canadiens français menaient pour préserver leur identité, La Presse a changé de point de vue et a commencé à appuyer les actions des Métis rebelles.

Mots-clefs: Les Métis. Louis Riel. La Presse. Fédération Canadienne. Semiosphère.

Abstract: The Métis rebellions of the North-West, guided by Louis Riel, changed Quebec's position in the Canadian federation. There was an awareness of the differences between Anglophones and Francophones that were not taken into account in the British North America Act. In this article, we analyze some editorial extracts of LA PRESSE, from February to November 1885, photocopied from microfilms in Quebec's University in Montreal. We see that the first editorials had a great symbiosis with the idea of the union within the federation. They condemned the "savagery" of the rebel Indians and Métis. The reaction of the readers, however, changed the newspaper's point of view. These readers considered the Rebels' fighting similar to that of French Canada, in order to preserve their cultural identity. The newspaper then followed the mind of its public and began to support the Rebels' cause.

Key words: The Métis. Louis Riel. La Presse. Canadian Federation. Semiosphere.

Beaucoup d'encre a déjà coulé sur les griefs des Canadiens français à l'égard du fédéralisme canadien. Rappelons brièvement que le Canada est une fédération de 10 provinces et de 2 territoires. Ces derniers, situés au Nord du pays, sont administrés directement par le gouvernement fédéral. Le Québec est la province la plus grande avec 
une superficie trois fois plus grande que celle de la France et qui correspond à 15\% de celle du Canada; sa population est de 8 millions d'habitants environ sur une population de 36 millions de Canadiens.

Le Québec intègre la région des Grands Lacs et du Saint-Laurent. La province est pratiquement jumelée avec la province adjacente de l'Ontario. Leurs économies sont intégrées, et forment le cœur financier, industriel et démographique du pays.

En 2017, il y a maintenant un siècle et demi que le Canada s'est doté de la structure fédérale qu'il a conservée jusqu'à nos jours. Les Canadiens français, comme le signale De La Rue (1963), sont entrés de bon gré dans la fédération, après avoir travaillé d'arrachepied pour sa mise en œuvre. De la sorte, vu son poids, le Québec aurait pu, dans le cadre de l'autonomie provinciale, s'administrer dans sa langue, sauvegarder et développer sa culture francophone sans grand problème.

La fédération canadienne a donc rendu possible la formation d'une entité administrative et territoriale disposant comme les autres provinces canadiennes d'attributs étatiques, et servant de base et de foyer à l'individualité ethnique, linguistique et culturelle Canadienne française ( DE LA RUE, 1963, p. 333).

Toutefois, face à tant de conflits et à la volonté de sécession du Québec, certains se sont demandés ce qui a mal fonctionné dans le fédéralisme canadien. De fait, le fédéralisme, comme dans le cas du Brésil et des USA, est un mode d'existence et d'organisation étatique qui vise à assurer l'unité de groupes nationaux divers et même antagonistes. L'essence du fédéralisme consiste à intégrer dans l'État les diversités ethniques et régionales. Il a pour but d'absorber et de neutraliser les frictions et les antagonismes qui, sans l'appareil fédéral, aboutiraient à des conflits radicaux susceptibles de désagréger l'ensemble.

J'aimerais avancer que les rébellions de l'Ouest canadien, à la fin du XIX ${ }^{\text {ème }}$ siècle, menées par Louis Riel, ont eu beaucoup d'influence sur la prise de conscience des Canadiens Français: ils ont compris qu'ils n'étaient pas traités sur le même pied d'égalité que leurs compatriotes ontariens. J'ai pu observer les changements de pensée à l'égard de l'Union canadienne, en investiguant les éditoriaux du journal La Presse, entre février et novembre 1885. Celui-ci avait vu le jour l'année précédente. Mais avant de poursuivre nos analyses des éditoriaux, quelques éclairages sur le fonctionnement des sémiosphères s'imposent. 


\section{Formation des sémiosphères}

Dans l'univers culturel désigné par la notion de sémiosphère ${ }^{2}$, il n'est plus question de systèmes de signes dans leurs structures dites immanentes. Les espaces, ruraux ou urbains, deviennent des lieux d'écriture, mais des lieux qui permettent l'affleurement de régions déterminées susceptibles de signifier la complexe diversité sémiotique qui caractérise la configuration spatiale des pays. On admet, dans un premier temps, qu'une région est un espace physique et palpable avec ses limites géographiques. La tension entre fragmentation et unité territoriale impose une visée privilégiée d'interrelations qui autorisent l'entrecroisement des foyers culturels spécifiques. Le discours fictionnel rend compte donc de territoires capables de mettre en relation des lieux variés de formations culturelles, de telle sorte que la perspective géographique, qui ne néglige pas, dans les études sur l'espace, les enchâssements des thèmes identitaires, est souvent assortie d'une confrontation entre certaines figures spatiales. Ces figures, formées d'espaces en transformation, troublent l'idée de frontière apte à penser les contours d'un centre sémiosphérique.

Dans une perspective littéraire, les textes autorisent une ouverture ou une clôture, des possibilités de passage, ou non, des figures périphériques vers les centres. Le souffle du mouvement peut être assumé par des sujets diachroniques ${ }^{3}$ ou des sujets épisodiques qui passent simplement sans chercher à laisser de traces ou à s'investir dans un parcours d'élancement entre le centre et la périphérie. La frontière pourra être, ou non, pour lui, un espace-charnière crucial, de contact culturel ${ }^{4}$. Mais l'importance de ce contact culturel devra être évaluée selon sa capacité plus ou moins ample à «gigogner» l'espace, dans des opérations appropriées pour permettre des syncrétismes de pensées et de cultures distinctes dans une même sémiosphère.

La nature imaginaire ou idéologique de la notion d'espaces de sémiosphères est particulièrement sensible dans les textes qui définissent la communication dans les espaces-charnières entre des cultures différenciées. Mais la réalité des espaces des sémiosphères semble sujette à caution. Il y a des passages et des juxtapositions qui finissent par se chevaucher, mais il y en a d'autres dont la nature se montre si complexe qu'il faut vraiment occulariser ${ }^{5}$ les déplacements des sujets diachroniques avec leurs disjonctions et conjonctions particulières. Pour ce faire, les exercices des sujets 
diachroniques et épisodiques sont parfois suivis par des narrateurs itinérants qui ont pour fonction d'assurer des degrés différenciés de relations entre représentation et réalité, de telle sorte qu'ils préparent les mouvements dynamiques de transformation des frontières.

\section{La scène communicative de la sémiosphère canadienne}

Comme nous l'avons déjà dit, nous observons la place que le Québec occupe dans la sémiosphère de la fédération canadienne par l'étude des éditoriaux du journal La Presse qui venait de naître, et qui se stabilisait comme périodique au Québec, dans l'interprétation des événements du Nord-ouest.

Remarquons d'emblée que tout centre de sémiosphère a besoin d'une autodescription avec un langage développé et structurellement organisé. La sémiosphère est traversée de langages variés qui circulent entre deux axes. Un axe horizontal qui représente la temporalité (passé, présent, futur), alors qu'un axe vertical indique l'espace (interne, frontière, externe). Ces différents langages appartenant à des temporalités et à des points spatiaux différents contribuent à faire de la sémiosphère un univers hétérogène en dépit de son apparence d'unité.

Le premier éditorial de La Presse, que nous observons, met en scène un énonciateur journalistique, dans la fonction du narrateur, apte à décrire la formation de l'axe central de la société québécoise.

\footnotetext{
Nous avons toute raison de croire que l'autorité du gouvernement sera complètement affirmée, et nous espérons de plus qu'il n'y aura plus d'effusion de sang.(...)

C'est la lutte antique de la civilisation contre la barbarie. Chaque fois que ces deux influences viennent en contact, le conflit est inévitable, et il y a effusion de sang.

Il en a été de même depuis le commencement du monde, le meurtre d'Abel. ${ }^{6}$
}

Il va s'intéresser aux troupes de Louis Riel, en soulignant qu'elles sont composées de trois variantes: les Sauvages-Métis, les Sauvages-Indiens et les Métis chasseurs. Le terme Sauvages, utilisé dans un sens large, intègre les Indiens et les Métis dans l'idéologème de la barbarie, qui est opposé à celui de la civilisation. Nous voyons maintenant apparaître le même réseau chronotopique de la confrontation historique millénaire qui habite la 
conjoncture de l'histoire américaine et mondiale. L'évocation du meurtre d'Abel nous rappelle son contraire, son frère Caïn, qui devient le symbole des rebelles qui menacent l'harmonie de l'axe central du Canada.

Le dernier énoncé s'appuie sur l'hypotexte biblique et sur ceux de la colonisation américaine marqués par la confrontation des colons et des dits sauvages; non seulement le journaliste sait qu'il doit en ce moment condamner ces guerriers exclus qui réclament leur insertion au sein de la sémiosphère canadienne récemment établie, mais encore il sait qu'il lui incombe de prononcer ces énoncés que l'histoire officielle lui dicte littéralement. Dans un second moment, le terme sauvages se différencie de celui de Métis et dénote simplement les Indiens. Les journalistes actualisent les énoncés codifiés par l'histoire particulière du Canada. Il s'agit là des opérations d'auto-description destinés à resserrer le codage des langages structurés de la sémiosphère canadienne.

Ces détails rappellent les anciens temps de notre histoire, où les Breboeuf, Jogues, Lallemand, succombèrent, victimes de la haine et de la barbarie.

De tous ces attristements, on ne peut obtenir qu'une consolation : le sang versé par les missionnaires a toujours été la semence la plus féconde, et a fait germer les plus éclatantes conversions. ${ }^{7}$

Les Sauvages se sont rendus coupables de meurtres horribles; ils ont tué des hommes, des femmes et des enfants innocents; ils ont versé le sang des missionnaires qui avaient voué leur existence à leur enseignement religieux et intellectuel. Il est nécessaire qu'ils soient punis comme ils le méritent. ${ }^{8}$

L'événement qui autorise cette dévalorisation des Indiens c'est la bataille du Lac à la Grenouille, au cours de laquelle ils ont été commandés par le chef Grand-Ours qui fut jugé par la suite et, après trois ans de prison, libéré; Esprit Errant, le chef guerrier des Cris fut pendu. L'argument barbarie vient s'ajouter aux idéologèmes du mal pour dresser le portrait empreint de sauvagerie d'individus qui agiraient dans le but d'anéantir la société, en tuant des innocents et les religieux intellectuels qui n'existeraient que pour promouvoir ladite société. Cet argument appartient encore à l'autodescription de la sémiosphère canadienne qui utilise les moteurs de sa force centrifuge pour expulser les dits «sauvages» du cœur de la nouvelle nation. 
Toutefois, un changement radical de positionnement va se produire entre les articles du début de l'insurrection et ceux rapportant la condamnation de Riel! Le 28 mai, La Presse reçut une lettre intitulée Plusieurs Lecteurs qui contestait le contenu d'un article précédent dans lequel le journal affirmait que Riel était coupable. La lettre ajoutait que le Métis avait beaucoup d'amis dans la province. C'était déjà une sorte de dialogue interactif qui prenait position sur les points de vue du journal au sujet des décisions des Métis de «rompre avec les autorités», même si nous avons déjà vu que le 1er juin, les Sauvages étaient encore sévèrement critiqués?

Un aspect très révélateur de la construction de la scène communicative est le dialogue entre les journaux et la discussion sur le fonctionnement des canaux de communication, ce qui est le propre des journalistes narrateurs, qui deviendront des sujets diachroniques, pour activer les forces (centripète et centrifuge), promptes à miner le codage langagier du centre sémiosphérique. S'ils doivent présenter l'histoire comme une histoire des réseaux de communication et des conditions de production de discours, ils deviennent alors de puissants instruments de formation, de l'opinion publique soit pour la manipuler, soit pour la secouer quand elle véhicule les idéologèmes d'un groupe déterminé qui devient néfaste pour le fonctionnement de la sémiosphère.

La Presse discute les informations diffusées par la Gazette Officielle (02-04) concernant la formation d'une commission pour régler les réclamations des Métis relatives aux questions territoriales. Selon le journal de Montréal, il fallait réellement recenser les Métis vivant en dehors du Manitoba pour s'assurer qu'ils n'allassent pas recevoir deux fois les acres de terre. Il s'agit d'une information à portée historique qui indique d'ailleurs le positionnement de La Presse québécoise au sujet de cette question qui affectait les Métis Canadiens-Français.

Toutefois, c'est le 3 avril que le dialogue inter-journalistique s'instaure dans toute son acuité. C'est le moment de critiquer sévèrement les journaux libéraux, notamment l'Électeur qui «désespère le pays», avec des données erronées.

Voici ce que nous lisons dans l'Électeur:

Il suffit de songer que 7.000 Métis et 34.000 Indiens sont disséminés sur ces vastes territoires du Nord-Ouest, dont l'étendue n'est pas de moins de 800 miles. Cette population est aguerrie, bien armée 
munie d'armes perfectionnées qu'elle manie avec une adresse étonnante. ${ }^{10}$

Sur la base du dernier recensement des Territoires du Nord-Ouest, La Presse montre qu'un chiffre réaliste serait celui de 2000 hommes d'armes au grand jamais ce que L'Électeur diffusait. Une autre information:

L'Électeur nous donne un renseignement très curieux, au sujet de cette guerre du Nord-Ouest:

Il est hors de doute maintenant qu'une surveillance sévère est exercée sur le service des dépêches. Aucune n'arrive sous nos yeux avant d'avoir été corrigée, altérée dans le but d'entretenir une fausse sécurité dans les âmes. ${ }^{11}$

Cette thématique de la censure des informations traverse la scène de la communication en temps de guerre et de conflit. L'important consiste à vérifier comment La Presse repère les fonctionnements de cette activité de surveillance à travers ses éditoriaux journalistiques, en établissant même à travers plusieurs autres éditions cette communication métadiscursive sur le fonctionnement du canal, les fils télégraphiques. Ce qui se donne alors comme texte, avec les nouvelles lois médiatiques de production et de circulation journalistique, c'est aussi la représentation du fonctionnement d'un grand appareil médiatique d'information au sein de la sémiosphère canadienne où les deux forces centripète et centrifuge sont en train de miner les langages de l'harmonie de la fédération. En d'autres mots, la représentation de la société sera élaborée par les moyens de communication qui désormais interprèteront les réalités historiques des grilles d'information de plus en plus élaborées grâce à certaines performances techniques. D'une certaine façon, ce sont les media qui devront former dorénavant la nature des sujets diachroniques.

Les journalistes sont étonnés et font preuve d'un manque de connaissance au sujet de l'ingérence du pouvoir sur la production du message («la haute main sur le télégraphe») dont La Presse était informée par le truchement d'un autre journal. Ces éditoriaux révèlent une représentation donnée en termes tels qu'elle précise et légifère le jeu de correspondances entre source d'information, canal et publics visés. Concrètement, cela signifie admettre le postulat selon lequel les messages référentiels produits en 
temps de crise politique sont manipulés par un pouvoir déterminé et que seul le véhicule d'information qui adopte le fonctionnement métadiscursif peut renverser les idéologèmes dégagés par les messages contrôlés. ${ }^{12}$

C'est comme cueillir l'information événementielle, de courte durée, à but immédiatement conatif, pour la lancer dans la toile historique de longue durée; là où le régime normatif du temps politique du centre sémiosphérique peut capter les traces d'opérations de représentation des nouvelles événementielles du temps révolutionnaire et transformer ces nouvelles en faits historiques propres à une communauté.

Quand on lit La Presse entre février et novembre 1885, on y perçoit bien les changements d'orientation. C'est un journal canadien-français qui démarre en condamnant les Sauvages et les Métis de la lignée de Champlain, ceux qui continuaient le travail des coureurs de bois et des colons français catholiques, et cette attitude provoque des réactions de la part de la population lectrice du Canada français. D’autant plus que le journal se mit à déclarer qu'il était le véhicule de communication de cette population. Mais les changements de points de vue attirent un public lecteur qui se fidélise de plus en plus. Le 6 avril, l'éditorial Notre Circulation annonçait la légitimité d'un journal de langue française pour transmettre des informations avec la même compétence que celle des journaux en langue anglaise. Cela avait attiré la confiance des Canadiens-Français, qui devinrent alors des fidèles du journal.

\footnotetext{
Notre Circulation

C'est avec un sentiment de légitime orgueil, que nous annonçons à nos lecteurs le succès sans précédent de La Presse. En moins de six mois, puisque le premier numéro de notre journal est daté du 20 0ctobre dernier, notre tirage s'est élevé à plus de 12.000 numéros par jour.

La Presse démontre ainsi l'inexactitude d'un fait assez généralement admis jusqu'à ce jour: que les Canadiens-Français ne lisent pas ou qu'ils ne lisent que des journaux anglais.

Tant que les Canadiens-Français n'ont pas eu, dans les journaux publiés en langue française, les nouvelles et les renseignements nécessaires, joints à une critique parfaitement indépendante des hommes et des actes publics, ils ont été les puiser là où ils étaient sûrs de trouver les informations voulues. ${ }^{13}$
} 
On parle encore de cette fidélité des lecteurs, mais c'est le 19 novembre, après la pendaison de Riel.

Opinion Publique

Nous n'avons jamais eu la prétention de diriger l'opinion publique, notre rôle plus modeste s'est toujours borné à représenter fidèlement les divers courants d'opinion qui se forment dans notre population. Nous croyons avoir rempli ce rôle consciencieusement jusqu'à ce jour, mais pour ce qui concerne le mouvement en faveur de Riel, nous en avons une preuve convaincante. Tous les jours, nous recevons de nombreuses lettres de félicitations les plus chaleureuses pour avoir été l'interprète fidèle des sentiments de la presqu'unanimité de nos compatriotes. En même temps, on nous conseille de persister dans la ligne de conduite ferme et indépendante que nous avons suivie jusqu'à ce jour.

Notre tirage s'est élevé de 12.119 qu'il était la semaine dernière, à 13.200. Sans doute, la vente au numéro s'est ressentie de l'excitation générale, mais tout considéré nous pouvons évaluer à plus de 600 , les nouveaux abonnés de cette semaine.

Cette expression de sympathie de la part du public pour le journal indépendant est la meilleure preuve que l'opinion désire être renseignée, qu'elle demande des faits et non des plaidoyers spéciaux.

La Presse, n'ayant d'ordres à recevoir de personne, n'est pas gênée pour exprimer sa pensée et n'est pas exposée, après avoir dénoncé violemment le procès de Regina, à être forcée de faire volte-face et de tenter une diversion avec la picote ou aucune autre calamité publique.

Nous continuerons à suivre notre programme: Parfaite indépendance, patriotisme et équité. ${ }^{14}$

Le journal discute de sa propre capacité à être fidèle aux événements et aux opinions, qui émergent selon plusieurs points de vue dans la sémiosphère. Le rôle de ce journal franco-canadien serait ainsi préservé en tant qu'activité de représentation du processus historique qui modèle des sujets diachroniques aptes à comprendre l'histoire de la société dans laquelle ils agissent. Cela parce que les lecteurs intervenaient en écrivant des lettres dans lesquelles ils manifestaient leur appui à la cause des FrancoMétis. C'est dire que le discours journalistique passait pour dépendre de l'installation de toute la scène de la communication, avec les récepteurs et leurs objets de représentation inclus. De toute manière, les récepteurs deviennent un signe d'action dans le processus communicationnel et la mise en place de cette situation encodage-décodage souligne le 
caractère communautaire de la représentation et de la signification. Plus précisément, cette relation de codification dialoguée est un processus par lequel l'information manifeste son ancrage dans un contexte de production/réception déterminé historiquement. Il apparaît ainsi que le journal devient une frontière, un espace-charnière, qu'il est fondamental pour s’opposer aux normes langagières imposées par le centre.

Les preuves irréfutables du fonctionnement de cette scène communicative sont les lettres defélicitations et l'augmentation du nombre d'exemplaires vendus. Ce sont les piliers qui assurent l'autonomie définitive du véhicule de communication dont le programme est l'indépendance, le patriotisme et l'équité. Pour continuer ce programme, La Presse ne cessera de demander à ses récepteurs de continuer à participer à la construction de la scène de la communication, c'est-à-dire de continuer à acheter le journal et d'en faire la promotion auprès des amis et des parents. La logique des discours journalistiques est ainsi liée aux problèmes d'existence de la communauté. Par la suite, La Presse entretiendra la section journalière Prières pour Riel, où l'on mentionnera les messes et les services religieux «pour le repos de l'âme du patriote martyr; on exposera le portrait du martyr dans les vitrines; on soulèvera des fonds pour la femme de Riel; on rapportera les émeutes provoquées par les orangistes au Canada français; on évoquera aussi, venant de tous les coins du Canada français les rassemblements de gens qui protestaient contre la pendaison de Riel et brûlaient l'effigie de sir Mac Donald.

Ne voilà-t-il pas que toutes les relations de construction discursive, destinées à la mise en place d'une formation langagière vraiment synchronisée avec le processus historique de l'époque, en phase avec la mobilisation de la société pour massacrer et anéantir ces exclus et exilés, reposent sur les relations avec les moyens de communication. C'est pourquoi dans le cycle canudien (sur Canudos au Brésil), les écrivains n'ont jamais lâché leurs «hommes-mot» destinés à montrer que la guerre eut lieu, parce que les populations urbaines, qui ne connaissaient pas les réalités des sertões, demandèrent un dénouement patriotique par la médiation des nouvelles.

La temporalité révolutionnaire, avec sa charge de rupture et de dé-filiation, que les insurrections précitées inaugurent, évolue sans doute vers un chronotope historique riche de nouvelles relations. La reconstruction d'une image positive des peuples métis est certes 
tardive, et dut attendre presque un siècle, mais elle est l'une des conséquences directes de ces insurrections qui exigèrent une légitimation des métissages du Nouveau-Monde.

Le fait en est que, issus de la révolte contre la situation de marginalité à laquelle les populations métisses étaient condamnées, ces chronotopes historiques de nouveaux affrontements contre les classes dominantes se révèlent être des formes privilégiées d'autoconnaissance, de re-filiation et de re-création d'un nouveau type historique. Nous avons alors affaire à une catégorie mythique dans le sens où elle est capable d'inspirer des imaginaires porteurs de mondes possibles, de croyances virtuelles, bref d'existences plurielles. C'est aussi une mythique épique qui exalte la lutte de tout un peuple pour qu'il existe justement comme peuple dans l'histoire: il s'agit d'activer la force centripète qui le conduira à l'axe central de la sémiosphère. Ne serait-ce que pour être reconnu comme peuple dans une histoire.

Mais ces leçons d'épopée américaine semblent rester inachevées, signalant probablement les éternels mouvements de re-création des êtres du Nouveau Monde. Les luttes métisses rejettent toute tentative de retour nostalgique vers les certitudes identitaires d'un passé lointain; en même temps, un retour aux genèses permet de conserver dynamiquement les traces culturelles des héritages et des traditions dans la valorisation du croisement des regards. Ce qui est un défi considérable. Par le jeu visible des correspondances et des oppositions, dans les diverses manifestations artistiques d'une société, on peut déceler la dynamique de l'hypertextualisation qui nous permet de retrouver le vivier au sein duquel ne cessent de dialoguer les textes fondamentaux de l'histoire.

\section{En guise de conclusion}

Quand Lotman présente la notion de frontière comme marquant la limite entre un espace considéré central et un espace extérieur périphérique, il souligne le fait que la frontière est l'élément qui facilite la communication entre deux cultures, voire deux systèmes de pensée. Dans ce contexte, l'action des sujets diachroniques capables de filtrer et de lier les expressions significatives des deux systèmes sémiosphériques est mise en relief. 
Ces sujets sont aptes à encoder/décoder les relations de signes susceptibles de guider l'autodescription de l'axe central d'une périphérie. La multiplication de sens possibles émane de l'action des sujets diachroniques qui laissent entendre que les textes qui configurent une sémiosphère possèdent plusieurs niveaux de signification.

Il nous a donc été possible d'observer comment les scénographies de la communication d'un journal naissant dans le Canada Français peuvent dévoiler la façon dont la société a pris conscience de ses contradictions dans la structure forgée d'une fédération.

Nous remarquons tout d'abord que le journal représente les scénographies historiques des conflits entre les Sauvages et les missionnaires. En ce sens, celui-ci s'attend à ce que le Canada français se sente à l'aise dans la nouvelle sémiosphère fédératrice du Canada. Les provinces fédérées ont le même objectif, celui d'anéantir l'ennemi commun, afin d'assurer l'harmonie de ce pays pacifique.

Il y a toutefois des forces qui agissent sur les sémiosphères et qui déterminent les distances entre les éléments des cultures concernées. L'espace interne de la sémiosphère est donc en continuel changement, ainsi qu'en processus d'autodescription et de structuration. Petit à petit, les influences extérieures les plus puissantes réussissent à atteindre le noyau et à modifier les pensées et les valeurs. Et quand les composantes se trouvent sous l'emprise d'une influence différente les éléments culturels se transforment et contribuent par un certain dynamisme contradictoire à l'intérieur de la sémiosphère. C'est ce qui est arrivé au Canada français qui a modifié ses formes d'autodescription, tout en prenant conscience des différences conflictuelles qui l'éloignent des autres provinces de la fédération.

Naturellement, beaucoup d'autres composantes viennent encore renforcer la cause de la souveraineté au Québec, mais les dialogues entre le journal naissant La Presse et ses lecteurs au sujet de la cause des Métis, constituent l'embryon du désir de séparation. 


\section{Références}

CUNHA, Euclides da. Hautes Terres. Paris: Metallié, 1997, (orig. 1902)

DE La Rue, Phillipe Aubert. Le Québec et l'avenir du Canada. Persée, vol. 28, n.4, 1963, pp. 330-346.

Éditoriaux de La presse. Montréal, de février à novembre 1885.

LAVALLÈE, Ronald. Tchipauk ou le chemin du loup. Paris: France-Loisirs, 1987.

LOTMAN, Youri. La sémiosphère. PULIM, Limoges, 1999.

SOUZA, Licia S. De. Figures Spatiales de Montreal. Une geopoétique urbaine interaméricaine. Montreal: Société des Écrivains, 2017.

SOUZA, Licia S. De. Figures Spatiales de Montréal. In: Interfaces Brésil/Canada, vol.15. n. 2., pp. 42-67, 2015.

SOUZA, Licia S. De. Mythologies du Métissage au Canada et au Brésil. Saarbrücken: Presses Académiques Francophones, 2012.

SOUZA, Licia S. De. Traversées geopoétiques: Métis des Sertões et de l'ouest canadien. Disponible sur le site $<$ http://www.anaisabecan2011.ufba.br/Arquivos/Souza-Licia1. pdf>. Consulté le 01-02-2017.

SOUZA, Licia S. de. Utopies américaines au Québec et au Brésil. Sainte-Foy: Les Presses de l’Université Laval, 2004. Coll. Américana.

SOUZA, Licia S. De. Représentation et idéologie: les téléromans au service de la publicité. Montréal : Ed. Balzac, coll. Univers du discours, 1994.

STRAWSON, Galen. A Fallacy of our Age. Time Literary Supplement, 15 Octobre 2004. Voir une version plus longue du même article: Against Narrativity. Ratio, XVII, $\mathrm{n}^{\circ} 4,2004$, p. 428-452.

\section{Notes}

1 Departamento de Estudos Literários, Universidade do Estado da Bahia, Salvador, Bahia, Brasil.

Pesquisadora do CNPq. liciasos@hotmail.com

2 Youri Lotman (1999) ne propose pas un modèle d'abstraction des phénomènes culturels, mais un processus de modélisation de la «réalité sémiotiqu » qui les sous-tend. La sémiosphère est l'ensemble de références d'une culture donnée, nécessaire à l'instauration des systèmes sémiotiques qui la composent. Elle est en interaction permanente avec ses parties. Elle précède et en même temps rend possible toute sémiosis en vigueur dans le cadre d'échanges culturels. Elle est l'unité de base de la signifiance humaine elle-même. Hors cet espace de référence, il ne peut y avoir ni communication ni signification..

3 Le "sujet diachronique" est celui qui cherchera à faire l'expérience de son identité et de celle de ses partenaires à partir d'une connaissance qui provient du passé, des traditions de la région. Strawson (2004) distingue deux sortes d'expérience psychologique du Moi, la première diachronique, la seconde épisodique, selon la place que chacune accorde à la continuité du Moi dans le temps. Les «esprits diachronique » se représentent naturellement leur Moi comme quelque chose qui existait dans le passé et existera dans le futur. Les «esprits épisodiques», se considérant comme un Moi au présent, ne se pensent pas comme une instance qui existait dans l'expérience passée et qui existera dans une expérience future. 
${ }^{4}$ Nous avons forgé les figures spatiales des « espaces-charnière et espaces-gigognes» dans une communication de l'ACFAS 2015, avec le titre : Figures spatiales de Montréal dans une perspective de géopoétique urbaine». L'espace-charnière permet le contact de cultures distinctes, il comporte une frontière, et cette limite territoriale assure le passage entre les territoires limitrophes. Le passage peut permettre une cohabitation qui ne se fait pas toujours dans les meilleures conditions, mais avec des conflits. Donc un espacegigogne serait celui qui contiendrait les propriétés d'autres espaces qui s'emboiteraient dans un nouvel espace et qui pourraient ouvrir des horizons et des frontières nouveaux. Le gigogne a la capacité de reconfigurer la sémiosphère. (SOUZA, 2015, disponible en $<$ https://periodicos.ufpel.edu.br/ojs2/index.php/interfaces/article/viewFile/7272/5096>. L'article a servi de base à la production d'un livre (SOUZA, 2017).

5 Terme de la narratologie cinématographique, forgé par François Jost. Il a trait à une façon de focaliser avec l'œil. (JOST apud SOUZA, 1994).

6 La Presse, Montréal, le 21 avril 1885.

7 La Presse, Montréal, le 21 avril 1885.

8 La Presse, Montréal, le 1er juin 1885.

9 Il faut lire dans le roman de Ronald Lavallée, Tchipayuk ou le chemin du loup, les récits des départs de troupes canadiennes. C'est comme deux peuples fourrés ensemble dans un train pour traverser un pays qu'ils ne connaissent pas, qu'ils trouvent ennuyeux lorsqu'ils traversent les paysages de pins et d'épinettes. En plus, les Canadiens français sont considérés des êtres inférieurs, méprisés par leurs compatriotes anglophones, astreints aux menus travaux lors des expéditions. Les anglophones sont toujours des officiers.

${ }^{10}$ La Presse, Montréal, le 3 avril 1885.

${ }^{11}$ La Presse, Montréal, le 3 avril 1885.

12 Nous avons mené quelques études comparées sur les œuvres fictives qui ont abordé la rébellion canadienne et la rébellion paysanne du sertão, dans le village de Canudos, au Brésil, à la fin du XIX ${ }^{\text {ème }}$ siècle. L'écrivain Euclides da Cunha avait démontré cela dans une œuvre littéraire et, dans un autre travail, Souza (2012), nous avions conclu que cette activité critique de l'auteur envers les médias se justifiait par le fait que c'était un phénomène nouveau au moment de la guerre, et qu'il n'avait pu être témoin des manipulations fréquentes des messages médiatiques au cours du $\mathrm{XX}^{\mathrm{e}}$ siècle.

${ }^{13}$ La Presse, Montréal, le 6 avril 1885.

${ }^{14}$ La Presse, Montréal, le 19 novembre 1885. 